\title{
ROTEM Analyses of Low Molecular heparin effects with In Vitro Induced Thrombocytopenia
}

\author{
Thomas Kander ${ }^{1,2}$, Ulf Schött ${ }^{1,2 *}$ \\ ${ }^{1}$ Department of Anesthesia and Intensive Care, Skane Universisty Hospital, Lund, 22185 Lund, Sweden \\ ${ }^{2}$ Institution of Clinical Sciences, Medical Faculty, Lund University, Sweden $\mid$
}

*Corresponding author: Ulf Schött, MD, PhD, Professor, Senior Consultant Anaesthetist

Received date: September 09, 2020; Accepted date: December 30, 2020; Published date: January 06,2021

Citation: Thomas Kander, Ulf Schött (2021) ROTEM Analyses of Low Molecular heparin effects with In Vitro Induced Thrombocytopenia. J Women Health Care Issues. J Women Health Care Issues, 4(1); Doi:10.31579/2642-9756/034

Copyright: @ 2021 Ulf Schött, This is an open access article distributed under the Creative Commons Attribution License, which permits unrestricted use, distribution, and reproduction in any medium, provided the original work is properly cited.

\begin{abstract}
Background: Thrombocytopenia is correlated to hemorrhagic complications in patients with low molecular weight heparin (LMWH) thromboprophylaxis.

Aims: The aims of our study were to investigate an experimentally induced in vitro thrombocytopenia and then adding 2 types of LMWHs in vitro. Our hypothesis was that a platelet depleted whole blood sample could reflect a stronger synergistic anticoagulative effect of in vitro added LMWH than in the non-manipulated blood.

Method: Two venous citrated blood samples were consecutively drawn from 8 patient's gynaecologic cancer and normal routine coagulation laboratory analyses immediately preoperatively. One of the two samples had its buffy coat pipetted away into a separate tube. Half of the buffy coat was returned to the same sample (treated sample). $3 \times 500 \mu$ of blood from the non-treated sample was added to 3 separate microtubes and corresponding for the treated sample. Thromboprophylactic doses corresponding to an in vivo peak effect 0.5 anti-Xa international units $/ \mathrm{ml}$ of tinzaparin and enoxaparin were added both to untreated and treated samples - 2 microtubes were unheparinized (treated/untreated sample). All samples were analysed with rotational thromboelastometry (ROTEM).

Results: Wilcoxon matched-pairs signed rank tests of the in-group differences between non-non-treated and treated samples showed no significant differences $(\mathrm{p} \leq 0.05)$ for any of the parameters analysed with the ROTEM-INTEM reagent regardless of heparinization or not. Calculation of non-parametric spearman correlation for clotting time (CT) vs. platelet count (PLC) were not significant for any group. Tinzaparin was clearly observed to prolong CT in the buffy-coat lowered blood from two patients.

Conclusions: Our results corroborate previous research that ROTEM cannot detect anticoagulative effects of low dose LMWH in patients with normal PLC. In two patients there was a clear prolongation of clot initiation after tinzaparin that warrants further studies on a more developed in vitro induced thrombocytopenia model.
\end{abstract}

Keywords: communicable disease; gynecology; pandemics; workflow

\section{Introduction:}

There is a need to address the dose of low-molecular-weight-heparins (LMWH) for venous thromboembolic (VTE) disease in thrombocytopenic patients, since evidence regarding safely administering LMWHs in the presence of different levels of thrombocytopenia is unclear.

Should the thromboprophylactic or therapeutic dose as in deep venous thrombosis (DVT) or pulmonary embolism (PE) treatments be maintained at the same dosage level as in non-thrombocytopenic patients, reduced or cancelled? The degree of thrombocytopenia and the strength of the plasma coagulation, often addressed as hypo- to hyper-coagulability needs to be addressed too. Low body weight $<50 \mathrm{~kg}$, renal failure with glomerular filtration rate $<30 \mathrm{~mL} / \mathrm{min} / 1.73 \mathrm{~m}^{2}$ or being elderly will all increase bleeding on LWMHs. Therefore, a weight-based approach with regular dosing assessments during platelet count (PLC) changes has been proposed [1].

PLC and function can change quickly in trauma, intensive care, and obstetrics and in massively bleeding patients. VTE disease has a high incidence in many of these conditions, but the optimal thromboprophylactic regimen has not been conclusively agreed on [2].

Recurrent thromboembolism, bleeding, and thrombocytopenia are frequently seen in cancer patients with cancer-associated thrombosis (CAT) [3], but guidelines for management have low evidence [4] and differ [5-9]. Cancer patients face great risk of succumbing to VTE, the second leading cause of death in their patient group and they are six times more likely than the general patient of developing it [3]. Patients with hematologic malignancies are at particular risk, since their oftenexperienced prolonged thrombocytopenic periods make therapeutic 
anticoagulation difficult [10]. Although compensatory hemostatic mechanisms may be active in many patients [11], the presence of thrombocytopenia increases the risk of excessive spontaneous or traumatic hemorrhage $[12,13]$. When coupled with the fact that conventional antithrombotic treatments tend to be comparatively ineffective for prevention or treatment of CAT [14-16], thrombocytopenia renders management of VTE in patients with cancer highly problematic. Indeed, major bleeding has been found to be not only a relatively frequent and severe complication but also an independent predictor of mortality in patients with anticoagulated cancer [17]. Still anticoagulation for the treatment of venous thromboembolism during periods of thrombocytopenia may be considered in patients with hematologic malignancies.

Campell et al. [10] described 13 cases of patients with hematologic malignancies that were therapeutically anticoagulated with either LMWH or unfractionated heparin (UFH) at PLCs $<50 \times 10^{9} / 1$. There was one bleeding event, World Health Organization grade 2, that was documented in a patient receiving enoxaparin dosed twice daily, resulting in an overall bleeding rate of $7,7 \%$ in this case series. All 13 patients were administered platelet transfusions during the periods of severe thrombocytopenia. International society of Thrombosis and Haemostasis (ISTH) [6] supports platelet transfusion when PLC is less than $<50 \times 10^{9} / 1$ if on full-dose anticoagulation for acute cancer-associated VTEs. This have been questioned by others who related their own local experience of having the option of not giving platelet transfusion in patients on full-dose anticoagulation down to a PLC ranging between 25 and $50 \times 10^{9} / 1[4,6]$. Babilonia et al. managed these patients with dalteparin 100 units $/ \mathrm{kg}$ subcutaneously once daily at PLC $<50 \times 10^{9} / 1$ and in patients with PLC $>50$ $\times 10^{9} / 1$ with dalteparin 200 units $/ \mathrm{kg} /$ day without increased bleeding or less effective thrombosis treatment/prophylaxis in the low PLC-group [4]. The Memorial Sloan Kettering Cancer Center (MSKCC) implemented the following guidelines in 2011 for cancer patients in need for LMWH: administer full dose enoxaparin for if the patient had a PLC $>50 \times 10^{9} /$, halving the dose of enoxaparin for PLC $>25-50 \times 10^{9} / 1$, and no anticoagulation if PLC were $<25 \times 10^{9} / 1$ [18]. Mantha et al. studied the safety and efficacy of the Memorial Sloan Kettering Cancer Center (MSKCC) guidelines in 99 patients with 140 thrombocytopenic episodes of 7 or more days. The enoxaparin dose was modified in 133 of the 140 episodes (95\%) according to the guidelines. Withdrawing or reducing the anticoagulant dose was not followed by events of recurrent VTE or major bleeding in the study [18].

Khanal et al. retrospectively studied 128 adult patients with hematologic malignancies who were diagnosed with DVT/PE (19) over 2 years. The risk of bleeding in a clinically significant manner for patients with PLC $<50 \times 10^{9} / 1$ was nonsignificantly higher $(11 \%$ vs $6 \%, \mathrm{P}=0.22)$ including major bleeding ( $6 \%$ vs $2 \%$ ), but no difference in effects on DVT progression.

Ibrahim et al. [20] stated that low-dose enoxaparin could be safely administered at a PLC in between 20-55 $\times 10^{9} / 1$ in BMT analysis of 26 bone marrow transplant patients.

Another concern is heparin-induced thrombocytopenia (HIT) in patients receiving LMWH. Real testing for the presence of heparin-platelet factor 4 antibodies (HPF4-Ab) and starting alternative anticoagulation when HIT is suspected is low in everyday clinics [21]. The risk of HIT with LMWH is lower than with UFH, but a baseline PLC is recommended from which the development of HIT could be hinted at.

Tests for monitoring LMWH's include anti-factor Xa (anti-FXa), activated partial thromboplastin time (aPTT) and thrombin generation (TGA). Despite LMWH's varying affinities for FXa and thrombin better being reflected by TGA [22], anti-FXa is the current gold standard in monitoring effect. Both tests are performed on platelet poor plasma and do not reflect the cellular contribution to in vivo hemostasis [23], that are better reflected with whole blood analyses.

Thromboelastometry with a whole blood viscoelastic test like ROTEM can improve perioperative and trauma bleeding management and can detect low PLC (24). A thromboplastin activated ROTEM (ROTEMINTEM) corresponds to the routine aPTT - and has the best sensitivity to detect heparin anticoagulants. However, in patients with normal PLC thromboprophylactic LMWH dosages usually do not prolong ROTEM clot initiation [25]. ROTEM-INTEM sensitivity to detect LMWH effects in thrombocytopenic patients on clot initiation and other aspects of clotting that can be detected by ROTEM, such as clot propagation and clot amplification have not been studied.

The aims of our study were to develop an experimentally in vitro induced thrombocytopenia by pipetting and subsequently halving the buffy coat of the blood sample (the fraction that contains platelets), then adding 2 different types of LMWHs in thromboprophylactic dosages and study their effects on ROTEM-INTEM.

Our hypothesis was that the buffy-coat lowered citrated whole blood sample could reflect a stronger synergistic anticoagulative effect of the in vitro added LMWH than in the non-manipulated blood (normal PLC). In a previous study, tinzaparin prolonged clot initiation more than enoxaparin with the higher therapeutic in vitro doses [22]. Would ROTEM-INTEM detect effects of thromboprophylactic tinzaparin better than enoxaparin?

\section{Material and Methods}

\section{Patients.}

The study was approved by the regional ethical board in Lund (registration number DNR 2017/636), and all patients included in the study gave written informed consent prior to inclusion. The study was conducted in compliance with the Declaration of Helsinki, and the manuscript was prepared according to the Strengthening the Reporting of Observational Studies in Epidemiology (STROBE) guidelines for observational studies. The following inclusion criteria were used: Patients age 18 or older, who were admitted to Department of Anaesthesia in Lund for brachytherapy of cervical cancer. Exclusion criteria: Patients with known coagulation disorders or ongoing anticoagulation medication.

\section{Sampling}

Venous blood were drawn from an indwelling brachial catheter after first blood being discarded. Blood was collected in 2x2,7 mL 0.109 M citrated tubes (BD Vacutainer Systems, Becton-Dickinson and Company, UK) for ROTEM analyses.

\section{In vitro anticoagulation with tinzaparin and enoxaparin}

An in vitro dose of $15 \mu \mathrm{l}$ of tinzaparin (Innohep ${ }^{\circledR} ; 10 \mathrm{IU} / \mathrm{ml}$ ) or enoxaparin (Klexane ${ }^{\circledR} ; 10 \mathrm{IU} / \mathrm{ml}$ ) was pipetted into separate $0,5 \mathrm{ml}$ citrated blood vials (in Eppendorf Safe-Lock tube). This would give an in vivo peak effect of a LMWH thromboprophylactic dose of 0.5 anti-Xa IU $/ \mathrm{ml}$ plasma (assuming that plasma accounted for $60 \%$ of the blood volume) [25], The samples were then incubated for 10 minutes at $37^{\circ} \mathrm{C}$ before ROTEM analyses.

\section{Buffy-coat pipetting to in vitro reduce platelet count}

The blood samples intended for lowering the PLC by pipetting the buffy coat were centrifuged within 10 minutes after sampling at $200 \mathrm{~g}$ at $20^{\circ} \mathrm{C}$ for 10 minutes to obtain the plasma, buffy coat fraction and erythrocyte fraction (see Figure 1). 


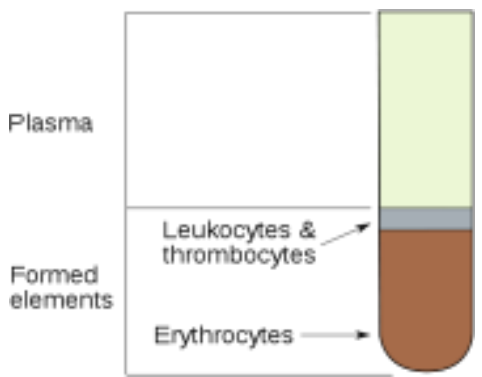

Figure 1: After centrifugation or after being allowed to stand in a test tube, erythrocytes settle as the bottom fraction of the samples contents. Leukocytes and platelets follow and make up most of the light-colored layer, known as the buffy coat, residing between the erythrocytes and the plasma. Leukocytes are denser than platelets and as such, platelets are found on top of the buffy coat
The buffy coat containing platelet and leukocyte fraction was pipetted away into a separate Eppendorf Safe-Lock tube and half of it returned to the $2,7 \mathrm{ml}$ citrate tube and then gently mixed with the plasma and erythrocytes. Then $0,5 \mathrm{ml}$ of this blood was pipetted into 2 Eppendorf Safe-Lock tubes.

\section{ROTEM}

Thromboelastometry (ROTEM, TEM International GmbH, Munich, Germany) was used to measure clot formation and clot elasticity. ROTEM has a fixed sample cup with a pin suspended in the blood sample. After the addition of $20 \mu \mathrm{l}$ of $0,2 \mathrm{M} \mathrm{CaCl} 2$ (Star-TEM) to $300 \mu \mathrm{l}$ of blood, coagulation was initiated by thromboplastin - INTEM reagent which better detects low levels of heparin and peak effects of thromboprophylactic and treatment dosages of LMWH [24,25]. The pin oscillates, and the movement is registered in the coagulating sample, which gives rise to a curve (see Figure 2).

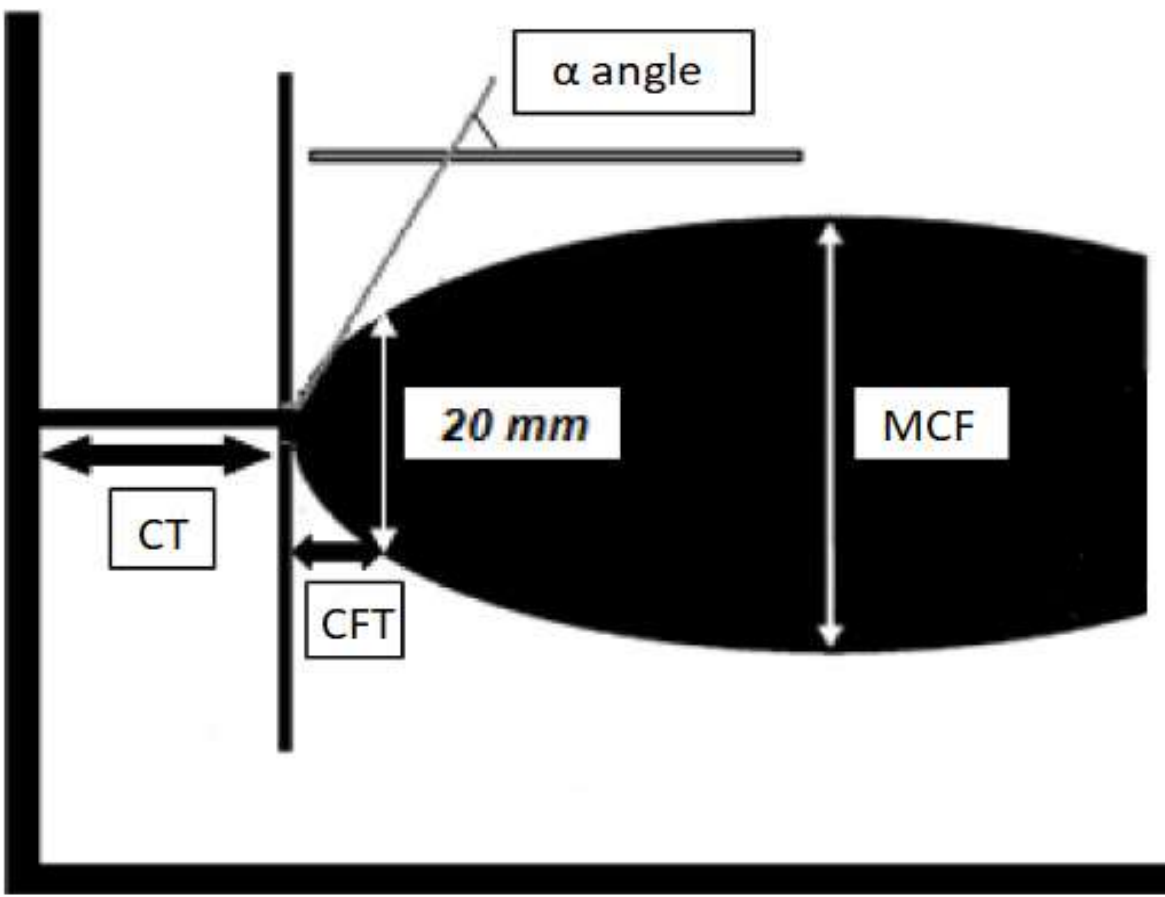

Figure 2: $\boldsymbol{C T}$ - Clotting time: The time it takes from the reagent has been added until the clot starts to form (clot initiation), reference range: $38-79 \mathrm{~s}$ with a coefficient of variation $(\mathrm{CV})$ of $<15 \%$.

CFT - Clot formation time: The time it takes for a clot to reach a firmness of $20 \mathrm{~mm}$ once the reagent is added, reference range: $34-159 \mathrm{~s} ; \mathrm{CV}<4 \%$. AA - Alpha Angle: The angle of the tangent of the curve at clot firmness $20 \mathrm{~mm}$, reference range: $63-83^{\circ} ; \mathrm{CV}<3 \%$.

MCF - Maximum clot firmness: reference range: $50-72 \mathrm{~mm} ; \mathrm{CV}<3 \%$.

\section{Several variables are obtained from the curve:}

CT: The time it takes from the reagent has been added until the clot starts to form (clot initiation), clotting time (CT), reference range: $38-79 \mathrm{~s}$ with a coefficient of variation $(\mathrm{CV})$ of $<15 \%$. Reference range with INTEM reagent: $100-240$ s.

CFT: The time it takes for a clot to reach a firmness of $20 \mathrm{~mm}$ once the reagent is added, Clot formation time (CFT), reference range: $34-159 \mathrm{~s}$; CV $<4 \%$. Reference range with INTEM reagent: 30-110s.

AA: The angle of the tangent of the curve at clot firmness $20 \mathrm{~mm}$, Alpha angle (AA), reference range: $63-83^{\circ} ; \mathrm{CV}<3 \%$. Reference range with INTEM reagent: $70-83^{\circ}$.
MCF: And the maximum clot firmness (MCF) reference range: $50-$ $72 \mathrm{~mm} ; \mathrm{CV}<3 \%$. Reference range with INTEM reagent: $50-72 \mathrm{~mm}$.

All samples were analyzed within 60 minutes of blood collection and stored in a heating block at $37^{\circ} \mathrm{C}$ until ROTEM analyses at $37^{\circ} \mathrm{C}$.

\section{Statistical analyses}

Excel was used to store acquired data. GraphPad Prism (GraphPad Software, La Jolla, CA) was used for statistical analyses, tables and diagrams of the acquired data. Spearman's rank correlation test was used to calculate correlations, with two tailed p-values and statistical significance set at $p<0,05$. Wilcoxon matched-pairs signed rank test was used to calculate if difference between sampling points were significant, significance set at $\mathrm{p}<0,05$. 


\section{Results}

\section{ROTEM test results}

Each patient's blood samples were analysed with ROTEM-INTEM. The
Results of the buffy coat-pipetted blood sample were compared with the results from the paired sample not subject to pipetting of the buffy coat. Graphs of the test results are found in Figures 3, 4 and 5 for nonheparinized, tinzaparinized and enoxaparinized blood, respectively.
Figure 3
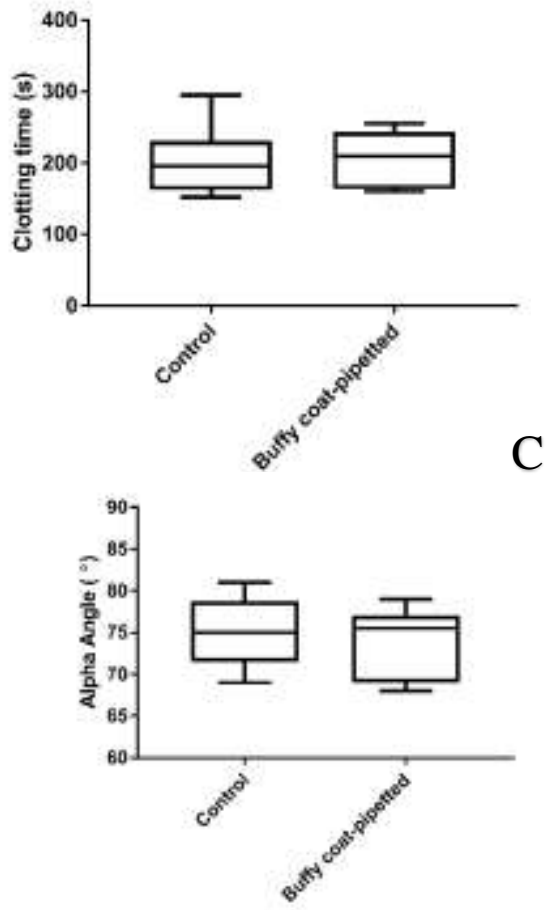

A
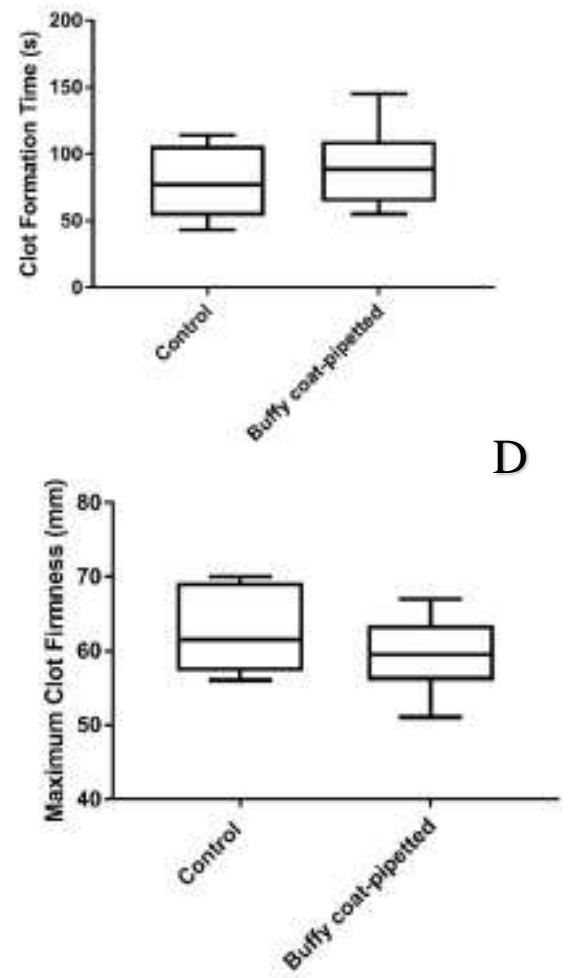

Figure 3a-d: Clotting Time (CT), Clot Formation Time (CFT), Alpha angle ( $\alpha$ ) and Maximum Clot Firmness (MCF) comparisons between 8 whole blood samples (labeled control) and their paired samples that were subject to our method of buffy coat reduction by pipetting. CT $p=0.9375$, CFT $p=0.1953, \alpha p=0.1484, M C F p=0.0625$.

\section{Clotting time}

Among the nonheparinized samples, the buffy coat-pipetted samples' measurements of CT had longer shortest CT, longer median, larger interquartile range, but shorter longest $\mathrm{CT}$ than the samples where the experimental method of reducing the buffy coat had not been performed (see Figure 3a). The tinzaparinized samples had a shortened median CT and increased spread in CT between samples in the buffy coat-pipetted samples compared with their paired control samples (see Figure 4a), while the enoxaparinized buffy coat-pipetted samples had a smaller interquartile range and shorter median CT compared with the enoxaparinized control samples (see Figure 5a). The differences between the samples in each group were tested with Wilcoxon matched-pairs signed rank test and were not found to be significant in any group.

\section{Clot formation time}

Measurements of CFT were longer in 7 of 8 nonheparinized buffy coatpipetted samples than their paired counterparts. The buffy coat-pipetted samples had a longer median CFT, a longer shortest CFT as well as the longest CFT measurement in their group (see Figure 3b). The tinzaparinized samples had larger spread above the $50^{\text {th }}$ percentile among the buffy coat-pipetted samples than the samples above the $50^{\text {th }}$ percentile that were not subject to buffy coat-pipetting (see Figure 4b). The measurements in enoxaparinized samples had larger spread among the buffy coat-pipetted samples, but a smaller interquartile range than the enoxaparinized control samples (see Figure 5b). Differences between samples in each group were tested with Wilcoxon matched-pairs signed rank test and were not found to be significant in any group.

\section{Alpha angle}

The rate of clot formation at clot firmness $20 \mathrm{~mm}$ was lowered in 7 of 8 buffy coat-pipetted samples in the nonheparinized group compared to their paired samples that did not have their buffy coat reduced (see Figure 3c). Lower results were found in the buffy coat-pipetted samples in 4 of the 7 pairs of the tinzaparinized group (see Figure $4 \mathbf{c}$ ). In the enoxaparinized group, on the other hand, 4 out of 7 pairs had larger alpha angle measurements in the buffy coat-pipetted samples than the samples not subject to our method of reducing the PLC in vitro (see Figure 5c). The in-group differences between controls and their paired samples were 
analysed with a Wilcoxon matched-pairs signed rank test and were not found to be significant.

\section{Maximum clot firmness}

The three groups differed in how many pairs had a different result in the buffy coat-pipetted sample compared to the paired control sample. In the nonheparinized group, MCF differed between control and buffy coat- pipetted in 7 of 8 pairs, 1 higher result in the buffy coat-pipetted sample and 6 lower (see Figure 3d). The tinzaparinized group had 5 lower measurements with the method and 2 higher (see Figure 4d). In buffy coat-pipetted samples in the enoxaparinized group there were 3 lower results and 4 higher results when comparing to their paired control samples (see Figure 5d). The in-group differences between controls and their paired samples were analysed with a Wilcoxon matched-pairs signed rank test and were not found to be significant.

Figure 4.
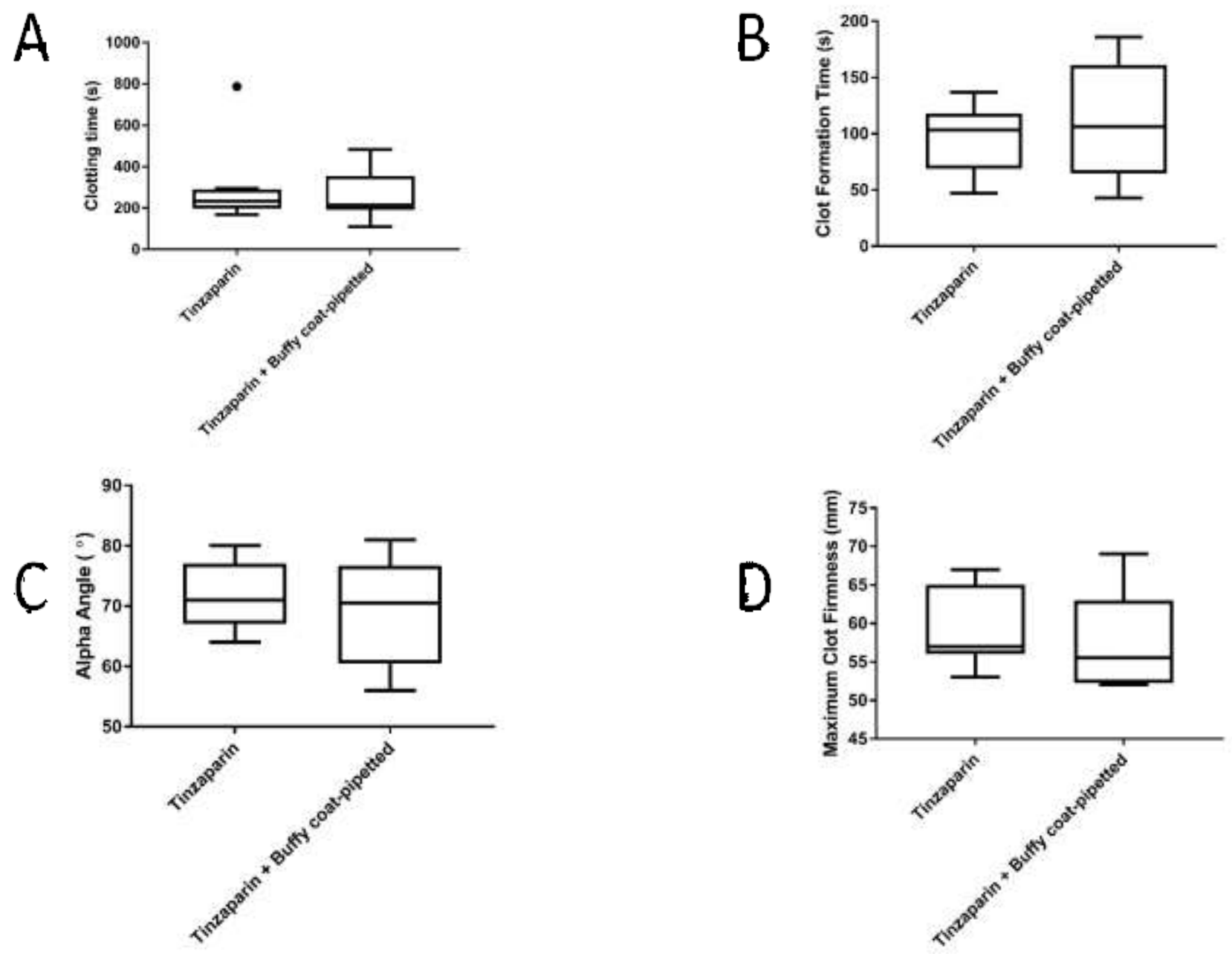

Figure 4a: Clotting Time (CT) comparison between 8 tinzaparinized whole blood samples and their paired samples that were subject to our method of buffy coat reduction by pipetting. CT $p=0.8125$.

Figure 4b-d: Clot Formation Time (CFT), Alpha angle ( $\alpha$ ) and Maximum Clot Firmness (MCF) comparisons between 7 tinzaparinised whole blood samples (labeled Tinzaparin) and their paired samples that were subject to our method of buffy coat reduction by pipetting.

CFT $p=0.5625, \alpha p=0.5625$, MCF $p=0.5469$. 


\section{Figure 5.}
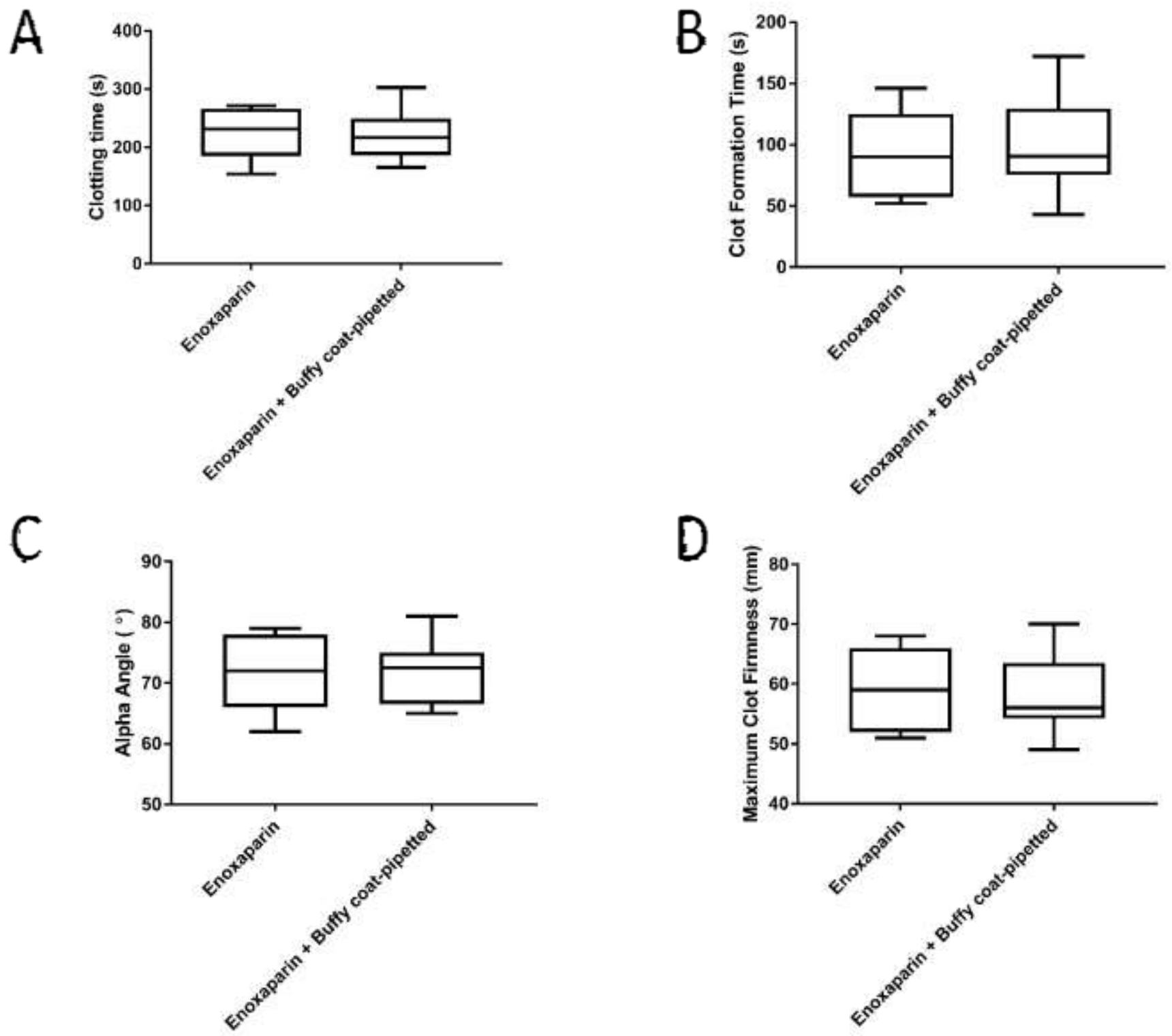

Figure 5a-d: Clotting Time (CT), Clot Formation Time (CFT), Alpha angle ( $\alpha$ ) and Maximum Clot Firmness (MCF) comparisons between 7 enoxaparinised whole blood samples (labeled enoxaparin) and their paired samples that were subject to our method of buffy coat reduction by pipetting. CT $p=0.5781$, CFT $p=0.9375, \alpha p>0.9999$, MCF $p=0.8750$.

\section{Correlation: platelet count and clotting time}

The samples' CTs were paired with their patients' PLCs recorded the same day and the samples that were subject to our method of reducing the PLC in vitro were assigned half of the earlier recorded PLC. A Spearman rank correlation between sample assigned PLC and CT was calculated for each group (see Figures 6a-c). For the nonheparinized group this resulted in $r_{s}=-0,6707$ for the control samples and $r_{s}=-0,5868$ in the buffy coatpipetted samples. The tinzaparinized samples had $r_{s}=-0,5946$ and $r_{s}=-$ 0,5509 and for the enoxaparinized samples the spearman rho was calculated to $r_{s}=-0,5225$ and $r_{s}=-0,5$ for control and buffy coat-pipetted samples respectively. 
Figure 6.

A

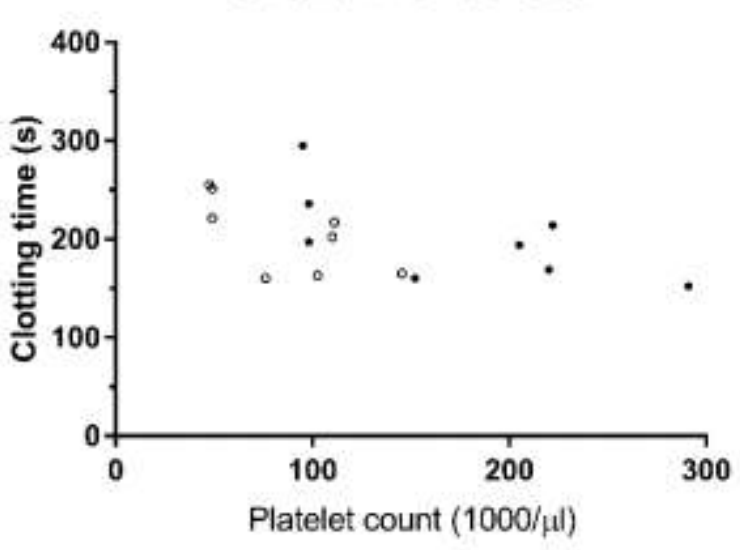

$\mathbf{B}^{-\quad \begin{array}{l}\text { Normal, spearman rho }=-0.6707 \\ \text { Buffy coat-pipetted, spearman rho }=-0.5868\end{array}}$

CT and PIc - tinzaparin

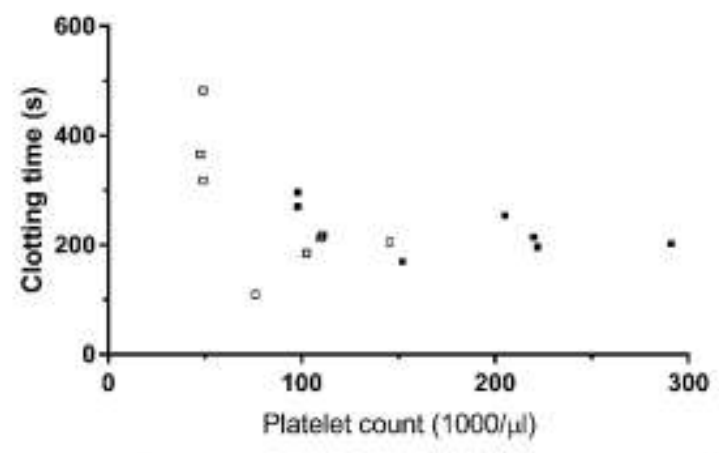

- Normal, spearman tho $=0.5946$

- Buffy coat-pipetted, spearman tho $=-0.5509$

\section{CT and Plc - enoxaparin}

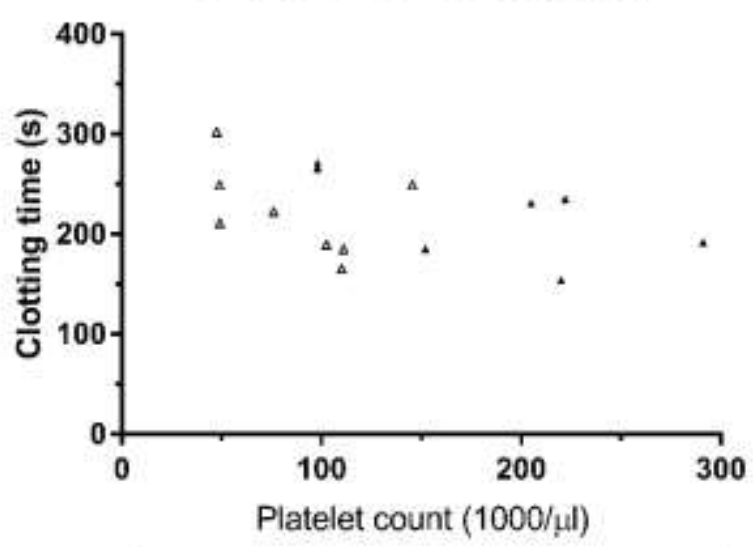

- Normal, spearman rho $=-0.5225$

4 Buffy coat-pipetted, spearman tho $=-0.5$
Figure 6: Clotting time (CT) as measured with ROTEM-INTEM and platelet count (PLC) as measured earlier that day. Buffy coatpipetted samples were assigned a PLC half of their paired sample.

6a: Non-parametric spearman rank correlation calculated for the nonheparinized samples to spearman rho $=-0,6707, \mathrm{p}=0,0765$ and spearman rho $=-0,5868, \mathrm{p}=0,1341$ for samples unheparinized and subject to buffy coat-pipetting.

6b: Spearman rank correlation calculated for the tinzaparinized samples to spearman rho $=-0,5946, \mathrm{p}=0,1730$ and spearman rho $=-$ $0,5509, \mathrm{p}=0,1625$ for samples tinzaparinized and subject to buffy coat-pipetting.

6c: Spearman rank correlation calculated for the enoxaparinized samples to spearman rho $=-0,5225, \mathrm{p}=0,2405$ and spearman rho $=-$ $0,5, p=0,2062$ for samples enoxaparinized and subject to buffy coat-pipetting. 
The correlations between the samples assigned PLCs and their CTs were not found to be statistically significant $(\mathrm{p} \leq 0.05)$ for any group. The control samples' correlations were calculated to $\mathrm{p}=0,0765, \mathrm{p}=0,1730$ and 0,2405 for the nonheparinized, the tinzaparinized and the enoxaparinized samples, respectively. The buffy coat-pipetted samples' correlations were calculated to $\mathrm{p}=0,1341, \mathrm{p}=0,1625$ and $\mathrm{p}=0,2062$ for the nonheparinized, the tinzaparinized and the enoxaparinized samples, respectively.

\section{Discussion}

It is of great interest to study the level of anticoagulant activity in both low-dose LWMH-thrombo-prophylaxis as platelet counts can change quickly in cancer and critically ill patients and it's not uncommon for them to have other complicating factors too, such as impaired kidney function that can increase bleeding complications [2,1]. This also holds for therapeutic dosages for treatments of PE and DVT.

However, there were no significant differences for any of the parameters analysed with the ROTEM-INTEM reagent regardless of heparinization with enoxaparin or tinzaparin or not at control platelet counts and at buffy coat-halved blood samples in our study. Calculation of non-parametric Spearman correlations for clotting time (CT) vs. control platelet counts (PLC) and simulated thrombocytopenia were not significant for anticoagulated or non-anticoagulated blood. Our hypothesis that the buffy-coat lowered citrated whole blood sample could reflect a stronger synergistic anticoagulative effect of the in vitro added LMWH than in the non-manipulated blood (normal PLC) is therefore rejected. However, two samples from patients with measured platelet counts beneath $100 \times 10^{9} / 1$ that were treated with buffy coat-pipetted had measured values of CT above the manufacturer's reference interval for ROTEM-INTEM. Earlier studies point to the importance of both platelets and fibrinogen [2,24] and our method should not have changed the levels of fibrinogen in the samples before ROTEM.

\section{Limitations}

A larger sample size would have been beneficial as it would have allowed for statistically more powersful analysis, making the inferrals more accurate by reducing the influence of varying, nonmeasured parameters affecting coagulation, like fibrinogen. The blood samples were not analysed for platelet counts after having had their buffy-coat halved. The correlation's validity rests on an assumption that any error in actual pipetted platelets would be insignificant in comparison to the variance in results in the analyses ran in the study design: ROTEM analysis of CT has a coefficient of variation of $<15 \%$.

We believe our results justify further studies. An improved method of inducing thrombocytopenia in vitro would make studies comparing LMWH monitoring in artificial thrombocytopenia in vitro with blood samples from thrombocytopenic patients possible. These studies can in turn, if artificial thrombocytopenia and native thrombocytopenia behaves similarly in analysis, provide means of studying the effects of thromboprophylaxis and of therapeutic dose regimes on thrombocytopenic blood with greater sample sizes. Greater samples sizes would reduce the influence that patients' differences in fibrinogen levels would have, as it's responsible for up to $50 \%$ of clot strength in thrombocytopenic blood [2]. ROTEM analyses at platelet counts $<100 \times 10^{9} / 1$ are also interesting as higher platelet counts do not influence ROTEM parameters, whereas fibrinogen does [24].
Conclusively, our results corroborate previous research that ROTEM cannot detect anticoagulative effects of low dose LMWH in patients with normal platelet counts. In two patients there was a clear prolongation of clot initiation after tinzaparin that warrants further studies on a more developed in vitro induced thrombocytopenia model.

\section{References}

1. Ibrahim RB, Skewes MD, Kuriakose P. 'Sailing in troubled waters': a review of the use of anticoagulation in adult cancer patients with thrombocytopenia. Blood Coagul Fibrinolysis. 2016;27(6):615-630.

2. Harr JN, Moore EE, Chin TL, Ghasabyan A, Gonzalez E, Wohlauer MV, Banerjee A, Silliman CC, Sauaia A. Platelets are dominant contributors to hypercoagulability after injury. J Trauma Acute Care Surg. 2013;74(3):756-762.

3. Donnellan E, Khorana AA. Cancer and Venous Thromboembolic Disease: A Review. Oncologist. 2017;22(2):199-207.

4. Reducing dose at trc Babilonia KM, Golightly LK, Gutman JA, Hassell KL, Kaiser JN, Kiser TH, Klem PM, Trujillo TC. Antithrombotic therapy in patients with thrombocytopenic cancer: outcomes associated with reduced-dose, low-molecularweight heparin during hospitalization. Clin Appl Thromb Hemost. 2014;20(8):799-806.

5. Babilonia KM, Golightly LK, Gutman JA, Hassell KL, Kaiser JN, Kiser TH, Klem PM, Trujillo TC. Antithrombotic therapy in patients with thrombocytopenic canceroutcomes associated with reduced-dose, low-molecular-weight heparin duringhospitalization. Clin Appl Thromb Hemost. 2014;20(8):799-806.

6. Farge D, Debourdeau P, Beckers M, Baglin C, Bauersachs RM, et al. International clinical practice guidelines for the treatment and prophylaxis of venous thromboembolism in patients with cancer. J Thromb Haemost. 2013;11(1):56-70.

7. Ibrahim RB, Skewes MD, Kuriakose P. 'Sailing in troubled waters': a review of the use of anticoagulation in adult cancer patients with thrombocytopenia. Blood Coagul Fibrinolysis. 2016;27(6):615-630.

8. Wagenhauser MU, Ertas N, Sagban TA, Witte M, Hoffman T, Schelzig H, Oberhuber A. A 61-year-old man with disseminated intravascular coagulation: a case report. Ann Vasc Surg 2014; 28:1566;17-22.

9. Easaw JC, Shea-Budgell MA, Wu CM, Czaykowski PM, Kassis $\mathrm{J}$, et al. Canadian consensus recommendations on the management ofvenous thromboembolism in patients with cancer. Part 2: treatment. Curr Oncol.2015;22(2):144-155.

10. Campbell PM, Ippoliti C, Parmar S. Safety of anticoagulation in thrombocytopenic patients with hematologic malignancies: A case series. J Oncol Pharm Pract. 2017;23(3):220-225.

11. Lewis JH, Burchenal JH, Ellison RR, et al. Studies of hemostatic mechanisms in leukemia and thrombocytopenia. Am J Clin Pathol. 1957;28(5):433-436.

12. James TN, Monto RW, Rebuck JW. Thrombocytopenia and abnormal bleeding in multiple myeloma. Ann Intern Med. 1953;39(6):1281-1287.

13. Rosenthal MC, Niemetz J, Wisch N. Hemorrhage and thromboses associated with neoplastic diseases. J Chron Dis. 1963;16(7):667675 . 
14. Edwards RL, Rickles FR, Moritz TE, et al. Abnormalities of blood coagulation tests in patients with cancer. Am J Clin Pathol. 1987;88(5):596-602.

15. Akl EA, Vasireddi SR, Gunukula S, et al. Anticoagulation for the initial treatment of thromboembolism in patients with cancer. Cochrane Database Syst Rev. 2011;(6):CD006649. doi 10.1002/14651858.CD006649.

16. Akl EA, Labedi N, Barba M, et al. Anticoagulation for the longterm treatment of thromboembolism in patients with cancer. Cochrane Database Syst Rev 2011;(6):CD006650. doi 10.1002/14651858.CD006650.pub3

17. Nieto JA, Solano R, Ruiz-Ribo' MD, et al. Fatal bleeding in patients receiving anticoagulant therapy for venous thromboembolism: findings from the RIETE Registry. J Thromb Haemost. 2010;8(6):1216-1222.

18. Mantha S, Miao Y, Wills J, Parameswaran R, Soff GA. Enoxaparin dose reduction for thrombocytopenia in patients with cancer: a quality assessment study. J Thromb Thrombolysis. 2017;43(4):514-518.

19. Khanal N, Bociek RG, Chen B, Vose JM, Armitage JO, Bierman PJ, Maness LJ,Lunning MA, Gundabolu K, Bhatt VR. Venous thromboembolism in patients with hematologic malignancy and thrombocytopenia. Am J Hematol. 2016;91(11):E468-E472.

20. Ibrahim RB, Peres E, Dansey R, Abidi MH, Abella EM, Gumma MM, Milan N, Smith DW, Heilbrun LK, Klein J. Safety of lowdose low-molecular-weight-heparins in thrombocytopenic stem cell transplantation patients: a case series and review of the literature. Bone Marrow Transplant. 2005;35(11):1071-1077.

21. ten Berg MJ, van den Bemt PM, Huisman A, Schobben AF, Egberts TC, van Solinge WW. Compliance with platelet count monitoring recommendations and management of possible heparin-induced thrombocytopenia in hospitalized patients receiving low-molecular-weight heparin. Ann Pharmacother. 2009;43(9):1405-1412.

22. Thomas O, Lybeck E, Strandberg K, Tynngård N, Schött U. Monitoring low molecular weight heparins at therapeutic levels: dose-responses of, and correlations and differences between aPTT, anti-factor Xa and thrombin generation assays. PLoS One. 2015 Jan 27;10(1):e0116835.

23. Schött U, Johansson PI. II. Bringing flow into haemostasis diagnostics. Br J Anaesth. 2013;111(6):864-867.

24. Larsson A, Tynngård N, Kander T, Bonnevier J, Schött U. Comparison of point-of-care hemostatic assays, routine coagulation tests, and outcome scores in critically ill patients. J Crit Care. 2015;30(5):1032-1038.

25. Thomas $\mathrm{O}$, Larsson $\mathrm{A}$, Tynngård $\mathrm{N}$, Schött U. Thromboelastometry versus free-oscillation rheometry and enoxaparin versus tinzaparin: an in-vitro study comparing two viscoelastic haemostatic tests' dose-responses to two low molecular weight heparins at the time of withdrawing epidural catheters from ten patients after major surgery. BMC Anesthesiol. 2015; $24: 170$.
This work is licensed under Creative Commons Attribution 4.0 License

To Submit Your Article Click Here: Submit Article

DOI:10.31579/2642-9756/034
Ready to submit your research? Choose Auctores and benefit from:

* fast, convenient online submission

* rigorous peer review by experienced research in your field

* rapid publication on acceptance

* authors retain copyrights

* unique DOI for all articles

* immediate, unrestricted online access

At Auctores, research is always in progress.

Learn more https://www.auctoresonline.org/journals/women-healthcare-and-issues 